\title{
Understanding reflectance anisotropy: Surface-state signatures and bulk-related features
}

\author{
W. G. Schmidt ${ }^{\text {a) }}$ and F. Bechstedt \\ Institut für Festkörpertheorie und Theoretische Optik, Friedrich-Schiller-Universität, Max-Wien-Platz, 1, \\ 07743 Jena, Germany \\ J. Bernholc \\ Department of Physics, North Carolina State University, Raleigh, North Carolina 27695-8202
}

(Received 17 January 2000; accepted 1 May 2000)

\begin{abstract}
Using a real-space multigrid method and ab initio pseudopotentials we calculated the reflectance anisotropy spectra for (001) growth structures of $\mathrm{GaP}$ and $\mathrm{InP}$ as well as for stepped $\mathrm{Si}(111): \mathrm{H}$ and hydrogenated $\mathrm{Si}(110)$ surfaces. Our results, obtained within DFT-LDA, show good qualitative agreement with recent experiments. Further improvement is achieved by using a numerically efficient GW approach with approximations for local-field effects and dynamical screening. We find the contributions to the anisotropy signal related either directly to surface states or to transitions between surface-perturbed bulk wave functions. Our results demonstrate the high sensitivity of RAS to the surface structure and chemistry and show that the optical transitions causing the anisotropy signal take place mainly in a few uppermost atomic layers of the substrate. (c) 2000 American Vacuum Society. [S0734-211X(00)04104-4]
\end{abstract}

\section{INTRODUCTION}

Optical spectroscopy has become an important tool of surface analysis in the last years due to its high sensitivity and in situ applicability. ${ }^{1}$ In particular, reflectance anisotropy spectroscopy (RAS), also called reflectance difference spectroscopy (RDS), is increasingly used to characterize static surfaces and to monitor dynamic surface processes in various environments. ${ }^{2,3}$ To fully exploit the technological potential of RAS, a theoretical understanding of the underlying physical mechanisms and reliable schemes to calculate RAS spectra are needed. The progress in the computational modeling of $\mathrm{RAS}^{4-6}$ has been rather slow, however. The agreement between the theoretical spectra, usually calculated by using slabs consisting of a few atomic layers, and the measured data is often unsatisfactory. This gave rise to suggestions that long-range effects, hardly accessible to slab calculations, are at least partially responsible for the surface optical anisotropy. A dynamic photon-induced localization of the initial and final states over the range of the penetration depth of light of several hundred $\AA$ was suggested to account for anisotropy signals close to the bulk critical point (CP) energies. ${ }^{7}$ In Ref. 8 another long-range effect, the quenching of bulk-state wave functions near the surface, was made responsible for the appearance of peaks at bulk CP energies in the surface spectra. These hypotheses are currently subject to both experimental ${ }^{9}$ and theoretical ${ }^{10}$ investigations. The present article aims at the understanding and interpretation of the surface optical anisotropy.

Realistic surfaces, and growth structures in particular, usually contain defects such as surface steps. The latter have been shown experimentally to give rise to distinct optical anisotropies, ${ }^{11-13}$ the origin of which is presently under de-

\footnotetext{
a) Author to whom correspondence should be addressed; electronic mail: W.G.Schmidt@ifto.physik.uni-jena.de
}

bate. The theoretical description of these step-related optical anisotropies is important for a correct interpretation of RAS spectra measured during growth and contributes also to a more complete understanding of the origin of RAS in general.

In the present work we combine converged calculations based on density functional theory (DFT) in the local density approximation (LDA) with a numerically efficient approximate GW approach or a scissors operator approach to calculate the reflectance anisotropy. As model systems we study the $(001)(2 \times 4)$ surfaces of $\mathrm{GaP}$ and $\mathrm{InP}$, steps on $\mathrm{Si}(111): \mathrm{H}$ surfaces as well as the hydrogenated $\mathrm{Si}(110)$ surface. We find good agreement with experiment. Both bulk-related ("intrinsic") and directly surface-state related ("extrinsic") contributions in the RAS are reproduced by well-converged slab calculations. This indicates that the difficulty in theoretical modeling of RAS is not conceptual, but rather caused by: (i) convergence problems due to the large numerical expense required for calculations of surface optical properties, and (ii) the difficulty in accounting for the many-particle effects in the spectra in an efficient yet accurate manner.

\section{METHODOLOGY}

Our DFT-LDA calculations are performed using firstprinciples pseudopotentials ${ }^{14}$ together with a real-space finite-difference method. ${ }^{15}$ A multigrid technique is used for convergence acceleration. The real-space method enables efficient parallelization and is thus particularly suited for the large supercells and many electronic states required for the calculation of the surface dielectric function. The electronic wave functions were mapped on a grid with a spacing of about $0.24 \AA$. To model the III-V(001) surfaces we used periodic supercells containing 12 atomic layers, the bottom 
layer saturated with pseudohydrogens, and a vacuum region equivalent to eight layers. The stepped $\mathrm{Si}(111): \mathrm{H}$ and $\mathrm{Si}(110): \mathrm{H}$ surfaces were described with symmetric slabs about $50 \AA$ thick, separated by $14 \AA$ of vacuum.

For the relaxation of the electronic and atomic degrees of freedom we sampled the surface Brillouin zone (SBZ) with four special $\mathbf{k}$ points in the irreducible part. The surface dielectric function was calculated with uniformly distributed $\mathbf{k}$ points corresponding to densities of 256 (GaP), 1024 (InP), and about 500 points $(\mathrm{Si})$ in the full $(1 \times 1)$ SBZ. Further details of the DFT-LDA calculations can be found in Refs. 15-18.

Calculations within DFT-LDA neglect the quasiparticle character of electrons and typically underestimate excitation energies, an effect known as the band-gap problem. ${ }^{19}$ For example, we calculate an indirect band gap of $0.58 \mathrm{eV}$ for $\mathrm{Si}$, while the room temperature experiment ${ }^{20}$ yields $1.11 \mathrm{eV}$. A similar underestimation of about $0.5 \mathrm{eV}$ occurs for the $E_{1}$ and $E_{2}$ CPs of the bulk band structure, for which we obtain 3.0 and $3.8 \mathrm{eV}$, respectively, and for the surface state energies of $\mathrm{Si}^{21,22}$ In order to compare the calculated RAS spectra for Si surfaces with experiment, we therefore apply an upward rigid shift of $0.5 \mathrm{eV}$ to the conduction bands and renormalize the momentum matrix elements accordingly. ${ }^{23}$ The same simple recipe has been followed in other recent $a b$ initio studies of the optical properties of Si surfaces. ${ }^{24,25}$

Such a rigid shift (or scissors operator approach) fails, however, if the underestimation of the excitation energies in DFT-LDA is less uniform than in Si. For bulk GaP, for example, it varies between 0.6 and $0.9 \mathrm{eV}$ for states close to the fundamental gap. Furthermore, as will be shown below, different shifts are obtained for bulk and surface state energies in the case of $\mathrm{GaP}$ and $\mathrm{InP}$. Rather than applying a rigid shift as for $\mathrm{Si}$ we therefore use $\mathrm{GW}$ calculations ${ }^{19}$ to take selfenergy effects into account for the III-V (001) surface. In order to obtain accurate excitation energies, the one-electron states should be obtained by solving the quasiparticle Schrödinger equation, i.e., the local exchange and correlation potential $V^{X C}(\mathbf{r})$ used in DFT-LDA should be replaced by the nonlocal and energy-dependent self-energy operator $\Sigma\left(\mathbf{r}, \mathbf{r}^{\prime} ; E\right)$ to describe the electron-electron interaction correctly. However, even in its lowest so-called GW approximation, where $\Sigma$ is expressed as a convolution of the singleparticle propagator $G$ and the dynamically screened Coulomb interaction $W$, its calculation remains a formidable task. In particular the large number of states entering the surface dielectric function makes calculations very expensive. Recently a linear parametrization of the quasiparticle shifts with respect to the surface localization of the corresponding electronic states was therefore suggested. ${ }^{26} \mathrm{We}$ used a different approach and introduced further approximations, following the schemes developed by Hybertsen and Louie $^{27}$ and Bechstedt et al. ${ }^{28}$ : In first order perturbation theory the GW quasiparticle energies are obtained from the DFT-LDA eigenvalues by

$$
E_{n, \mathbf{k}}=E_{n, \mathbf{k}}^{\mathrm{LDA}}+\frac{1}{1+\beta_{n, \mathbf{k}}}\left\{\Sigma_{n, \mathbf{k}}^{\mathrm{st}}+\Sigma_{n, \mathbf{k}}^{\mathrm{dyn}}\left(E_{n, \mathbf{k}}^{\mathrm{LDA}}\right)-V_{n, \mathbf{k}}^{X C}\right\},
$$

where the self-energy operator $\Sigma$ has been divided into static and dynamic contributions. $\beta_{n, \mathbf{k}}$ is the linear term of the expansion of $\Sigma^{\text {dyn }}$ in energy around the DFT-LDA eigenvalue $E_{n, \mathbf{k}}^{\mathrm{LDA}}$. The static part can be further divided into two parts:

$$
\begin{aligned}
\sum^{\mathrm{st}}\left(\mathbf{r}, \mathbf{r}^{\prime}\right)= & \frac{1}{2} \sum_{n, \mathbf{k}} \psi_{n, \mathbf{k}}(\mathbf{r}) \psi_{n, \mathbf{k}}^{*}\left(\mathbf{r}^{\prime}\right)\left\{W\left(\mathbf{r}, \mathbf{r}^{\prime} ; 0\right)-v\left(\mathbf{r}-\mathbf{r}^{\prime}\right)\right\} \\
& -\sum_{v, \mathbf{k}} \psi_{v, \mathbf{k}}(\mathbf{r}) \psi_{v, \mathbf{k}}^{*}\left(\mathbf{r}^{\prime}\right) W\left(\mathbf{r}, \mathbf{r}^{\prime} ; 0\right)
\end{aligned}
$$

representing the Coulomb hole $\Sigma_{\mathrm{COH}}$ and the screened exchange $\Sigma_{\text {SEX }}$. The $\psi_{n, \mathbf{k}}$ are the DFT-LDA wave functions and $W\left(\mathbf{r}, \mathbf{r}^{\prime} ; 0\right)$ and $v\left(\mathbf{r}-\mathbf{r}^{\prime}\right)$ denote the statically screened and bare Coulomb potentials, respectively (see, e.g., Ref. 19 for a comprehensive review). For the practical calculation of $W$ we model the dielectric function as ${ }^{28}$

$$
\epsilon(q)=1+\left\{\left(\epsilon_{\infty}-1\right)^{-1}+\left(q / q_{\mathrm{TF}}\right)^{2}+3 q^{4} /\left(4 k_{F}^{2} q_{\mathrm{TF}}^{2}\right)\right\}^{-1},
$$

where $k_{F}$ and $q_{\mathrm{TF}}$ represent the Fermi and Thomas-Fermi wave vectors, respectively. This expression interpolates between the correct behaviors at high and low $q$ vectors and by construction correctly obtains the static dielectric constant for $q=0$. This model reproduces very well the random phase approximation results for semiconductors. ${ }^{29}$ Using the LDA for $W,{ }^{27} \Sigma_{\mathrm{COH}}$ can now be calculated analytically, ${ }^{28}$ resulting in a potential that depends on the local charge density. For the calculation of $\Sigma_{\text {SEX }}$ we retain only diagonal elements in the Fourier transform of $W$. The local fields are included, however, by using state-dependent charge densities

$$
\rho_{n, \mathbf{k}}=\int d \mathbf{r}^{3} \rho(\mathbf{r})\left|\psi_{n, \mathbf{k}}(\mathbf{r})\right|^{2},
$$

in the calculation of $k_{F}$ and $q_{\mathrm{TF}}$. The dynamic terms $\beta_{n, \mathbf{k}}$ and $\Sigma^{\text {dyn }}$ in Eq. (1) can be approximated by simple integrals of the dielectric function. ${ }^{28}$ For the practical calculation we use Eq. (3) together with a single plasmon pole approximation to describe the frequency dependence. Local-field effects are again included using the mean-density approximation, Eq. (4).

After applying this simplified GW approach to the DFTLDA bulk band structure of $\mathrm{GaP}$ we obtain interband transition energies of 2.7 and $4.8 \mathrm{eV}$ for $E_{0}$ and $E_{0}^{\prime}$, respectively. $E\left(X_{1 c}\right)$ and $E\left(X_{3 c}\right)$ are shifted to 2.2 and $2.4 \mathrm{eV}$. In the case of bulk InP, the $E_{0}$ gap at $\Gamma$ changes from $0.9 \mathrm{eV}$ in DFTLDA to $1.4 \mathrm{eV}$. The transition energies $E_{1}$ and $E_{0}^{\prime}$ are shifted from 2.5 to $3.2 \mathrm{eV}$ and from 4.2 to $5.0 \mathrm{eV}$, respectively. These values agree with the experiment within $0.2 \mathrm{eV}$. The application of this GW scheme to the surface seems problematic, since the model requires the input of the dielectric constant $\epsilon_{\infty}$. Northrup ${ }^{21}$ and Rohlfing et al. ${ }^{22}$ have shown, however, that the inaccuracies caused by the use of bulk dielectric constants for surface GW calculations are very small. 

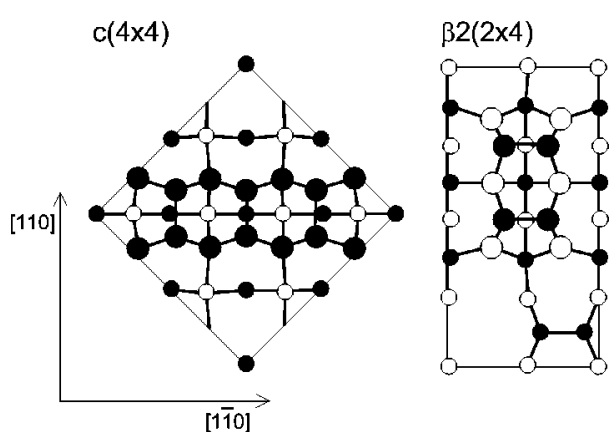

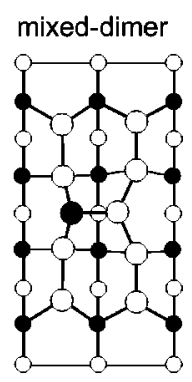

FIG. 1. Top view of $\operatorname{GaP}(001)(2 \times 4)$ surface reconstruction models. The structures are ordered according to their stoichiometry. Empty (filled) circles represent $\mathrm{Ga}(\mathrm{P})$ atoms. Large (small) symbols indicate positions in the first and second (third and fourth) atomic layers.
Finally, we use the electronic structure to determine the reflectance anisotropy from the slab polarizability according to the formalism developed by Del Sole $^{30}$ and Manghi et al. $^{31}$

\section{RESULTS}

\section{A. $\operatorname{GaP}(001)(2 \times 4)$}

Total-energy calculations ${ }^{17,32,33}$ for $\mathrm{GaP}(001)$ predict a sequence of $(2 \times 4)$ single-dimer reconstructions for cationrich surfaces. $\beta 2(2 \times 4)$ and $c(4 \times 4)$ reconstructions, known from $\mathrm{GaAs}(001)$, form for more P-rich surfaces. A top view of the GaP surface models is given in Fig. 1. These theoretical findings agree with the recent experiments, as discussed in detail in Refs. 17 and 33.

In Fig. 2 we show the RAS spectra calculated within DFT-LDA for the four $(2 \times 4)$ surface structures shown in Fig. 1. The top Ga dimer and mixed-dimer structures show a pronounced negative anisotropy in the low energy region, with minima (denoted $S$ in Fig. 2) between 2.0 and $2.3 \mathrm{eV}$. The strength of this anisotropy is directly correlated with the number of $\mathrm{Ga}-\mathrm{Ga}$ bonds along the [110] direction. Its magnitude is the highest for the top Ga dimer model with eight bonds, is slightly reduced, and shifted to lower energies for the mixed-dimer geometry with six cation-cation bonds, and is flattened for the $\delta$ structure with only two such bonds. The calculated spectra also show a strong dependence on the structural details at higher energies. For the $\beta 2$ geometry with three $\mathrm{P}-\mathrm{P}$ dimers oriented along [110], we find a relatively broad positive anisotropy between about 2.4 and 4.4 $\mathrm{eV}$. Maxima of the anisotropy appear around 3.2 and $4.1 \mathrm{eV}$ and a shoulder exists at $2.8 \mathrm{eV}$. The shape of this anisotropy is roughly preserved for the $\delta$ structure, which features one $\mathrm{P}-\mathrm{P}$ dimer. Its magnitude is, however, somewhat reduced and the spectrum is shifted downwards. An even further reduction in positive anisotropy occurs for the mixed-dimer and top $\mathrm{Ga}$ dimer structures, featuring single $\mathrm{Ga}-\mathrm{P}$ or $\mathrm{Ga}-\mathrm{Ga}$ dimers, respectively, on top of a Ga-terminated substrate. The evolution of the spectra in the high energy region shows thus a correlation between the positive anisotropy and the formation of $\mathrm{P}-\mathrm{P}$ dimers.

Our calculations are in accord with the available experimental data. ${ }^{17,34,35}$ The RAS spectra measured for the Garich phase of $\mathrm{GaP}(001)(2 \times 4)$ feature a strong negative peak around $2.4 \mathrm{eV}$. Both the top Ga dimer model and the mixed- dimer model are thus plausible candidates to explain the Garich surface phase. The measured spectrum for the Ga-rich phase shows a maximum between the energies of the $E_{0}$ and $E_{1}$ CPs. This peak, which should be observed between 2.1 and $2.9 \mathrm{eV}$ in the calculated spectrum, is absent in case of the top Ga dimer structure. It appears, however, as a weak shoul$\operatorname{der}$ (denoted $A$ in Fig. 2) for the mixed-dimer model. As will be discussed below, the agreement between the calculated spectrum for the mixed-dimer structure and the measured RAS improves substantially upon inclusion of self-energy effects, in particular with respect to the shoulder $A$. The RAS spectra thus indicate that the Ga-rich phase of $\mathrm{GaP}(001)(2$ $\times 4)$ corresponds to the mixed-dimer geometry. This assignment is corroborated by the recently measured surface corelevel shifts. ${ }^{36}$ The spectroscopic results indicate that the extreme Ga-rich limit, which according to the total-energy calculations ${ }^{17,33}$ is characterized by the top Ga dimer model, may actually not be reached experimentally.

The measured spectrum for the less Ga-rich $(2 \times 4)$ surface phase with an approximately balanced surface stoichiometry is dominated by a "camelback" spectrum with maxima between the energies of the $E_{0}$ and $E_{1}$ CPs and at $E_{0}^{\prime} \cdot{ }^{17}$ No negative anisotropy appears. The only computed spectrum with no (or very little) negative anisotropy belongs to the $\beta 2(2 \times 4)$ structure. Maxima appear at 3.2 and $4.1 \mathrm{eV}$,

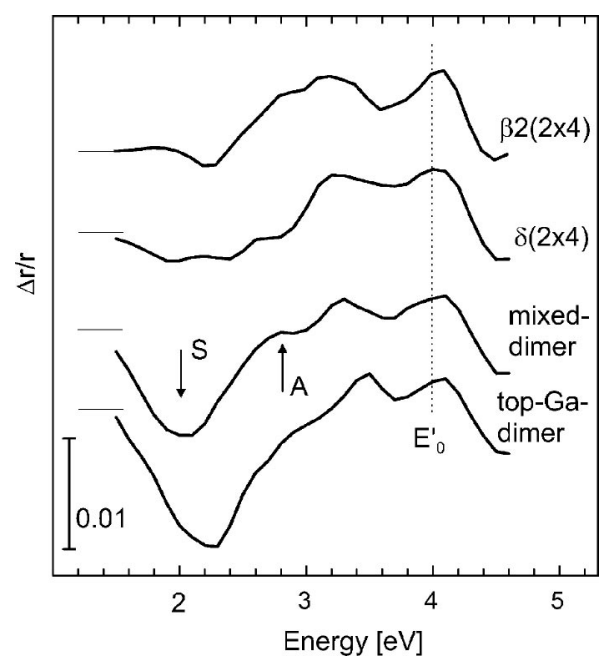

FIG. 2. RAS spectra $\left[\operatorname{Re}\left\{\left(r_{[\overline{1} 10]}-r_{[110]}\right) /<r>\right\}\right]$ calculated within DFTLDA for the four $\mathrm{GaP}(001)(2 \times 4)$ surface reconstruction models shown in Fig. 1. The calculated position of $E_{0}^{\prime}$ is indicated. 


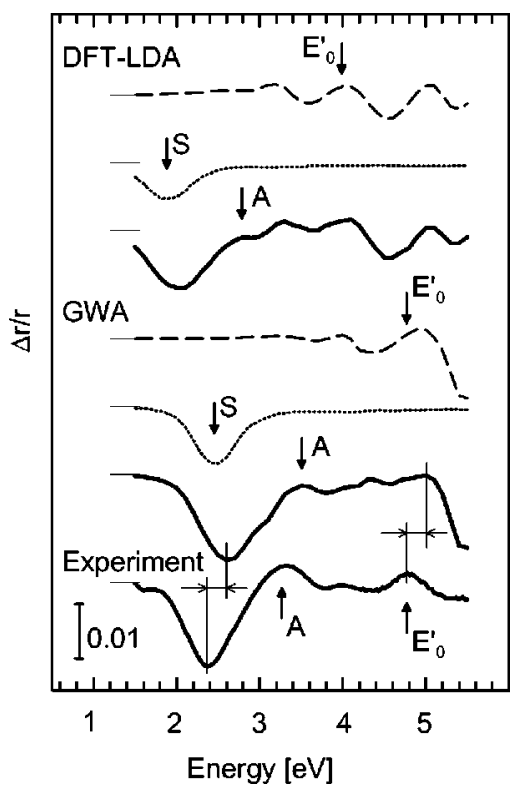

FIG. 3. RAS spectra $\left[\operatorname{Re}\left\{\left(r_{[110]}-r_{[110]}\right) /<r>\right\}\right]$ calculated within DFTLDA/GWA for the mixed-dimer structure are compared with the measured data (Ref. 17) for the Ga-rich $\operatorname{GaP}(001)(2 \times 4)$ surface. The calculated/ measured positions of $E_{0}^{\prime}$ are indicated. Dashed/dotted lines show the contributions to the RAS from transitions between subsurface/surface electronic states. Solid lines represent the calculations for the complete slab.

close to the calculated energies of the $E_{1}$ and $E_{0}^{\prime}$ CPs. Our results thus indicate that the less Ga-rich phase of $\mathrm{GaP}(001)$ $(2 \times 4)$ corresponds to the $\beta 2(2 \times 4)$ structure.

In the following, we focus on the Ga-rich surface phase, which corresponds to the mixed-dimer structure as discussed above. This structure is reproducibly obtained in experiments. ${ }^{17,35,36}$ To determine the origin of the optical anisotropy we spatially separated the contributions to the RAS from different slab regions. The dashed curves in Fig. 3 show the anisotropy due to transitions between the electronic states localized below the top four atomic layers. It is obvious that the surface modification of these bulk-like wave functions gives rise to strong anisotropies for high photon energies. In particular, a feature occurs near the calculated energy of the $E_{0}^{\prime} \mathrm{CP}$ of the GaP bulk band structure. It de- pends only weakly on the actual surface structure and is present in the calculated spectra of all structures included in our study (cf. Fig. 2). Experimentally, the maximum at the $E_{0}^{\prime} \mathrm{CP}$ was found irrespective of the surface preparation conditions. ${ }^{17,35}$

On the other hand, strong anisotropies in the low-energy part of the spectrum arise from transitions between surface localized states. In Fig. 4 we show the surface bands of the mixed-dimer model calculated in DFT-LDA, together with their orbital character. The highest occupied surface state, $V 1$, slightly above the bulk valence band maximum (VBM), corresponds to the $\mathrm{P}$ dangling bond at the mixed dimer. $V 2$ and $V 3$, slightly below the VBM, are related to $\sigma$ bonds between surface $\mathrm{Ga}$ atoms. $C 1-C 5$, in the upper part of the bulk band gap, arise from dangling bonds at the second-layer surface cations. [A detailed discussion of the $\mathrm{GaP}(001)$ surface electronic structure is given in Ref. 33.] In particular, transitions between $\mathrm{Ga}-\mathrm{Ga}$ bond-related states and empty $\mathrm{Ga}$ dangling bonds cause the strong negative anisotropy around $2 \mathrm{eV}$. The dotted lines in Fig. 3 show the RAS induced exclusively by transitions between the valence states $V 1, V 2$ and $V 3$ and the conduction states $C 1-C 5$. These eight states nearly completely account for the negative anisotropy observed at low photon energies. The assignment of cationrelated surface states to the RAS around $2 \mathrm{eV}$ corroborates the phenomenological link established by comparing the RAS of different structures as discussed above.

The optical anisotropy calculated for the complete slab (solid lines in Fig. 3) corresponds roughly to a superposition of the bulk-related features and surface peaks discussed above. This is a theoretical confirmation of the early work by Aspnes and Studna, ${ }^{37}$ who discriminated between two components of the reflectance anisotropy: "intrinsic" contributions from surface effects on bulk wave functions and "extrinsic" contributions related to the surface structure.

In Fig. 3 two sets of theoretical data are presented, obtained in DFT-LDA and GWA. As expected from the shifts calculated for the $\mathrm{GaP}$ bulk band structure, we observe a distinct blueshift of the spectrum upon inclusion of selfenergy effects. However, the shift is nonuniform: it amounts to about $0.6 \mathrm{eV}$ for the surface-state related features (dotted

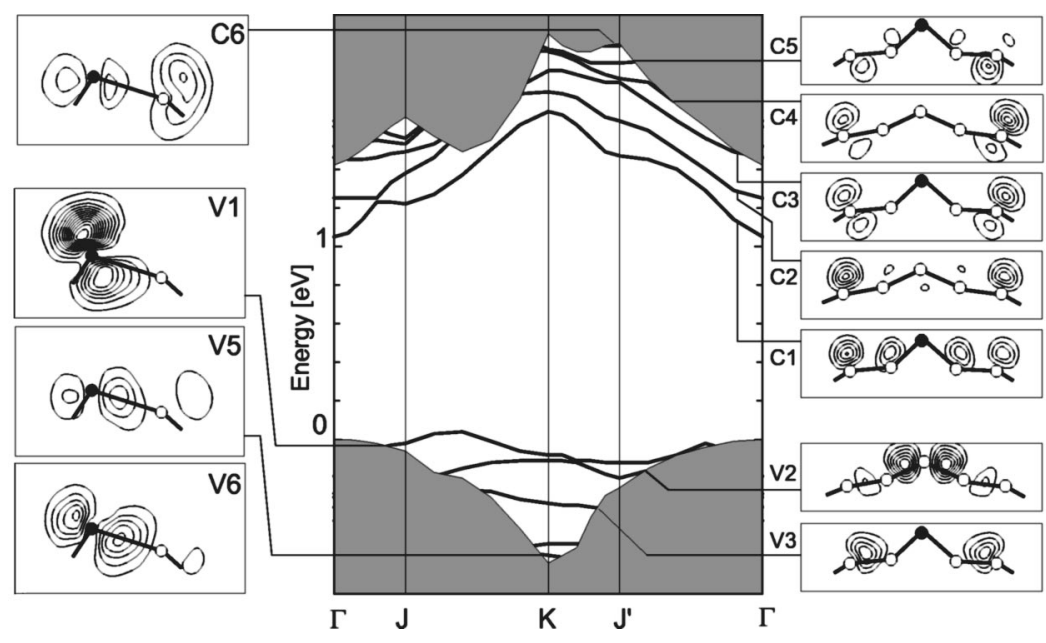

FIG. 4. Surface band structure (bound states) calculated within DFT-LDA for the mixed-dimer structure of $\mathrm{GaP}(001)$. Gray regions indicate the projected bulk band structure. In the left and right panels the corresponding squared wave functions at $K$ are shown. The contour spacing is $10^{-3} \mathrm{bohr}^{-3}$. 
lines) and $0.8-1.0 \mathrm{eV}$ for the bulk-related ones (dashed lines). The nonuniformity of the calculated quasiparticle shifts agrees with experiment: As indicated by the arrows in the lower part of Fig. 3, the energy shift between the measured data and the spectrum calculated in GWA is nearly constant and amounts to about $0.3 \mathrm{eV}$. The inclusion of selfenergy effects in the calculations not only leads to energy shifts, but also to pronounced changes in the line shape. The weak shoulder $A$ at $2.8 \mathrm{eV}$ in the DFT-LDA spectrum develops into a maximum at $3.5 \mathrm{eV}$, in good agreement with experiment.

While the agreement between the measured and the calculated spectra improves much upon inclusion of GW corrections, there remain discrepancies: the excitation energies are somewhat overestimated and the line shapes, in particular for higher energies, do not agree very well. This is not too surprising: one limitation of our study is the neglect of electron-hole interactions. ${ }^{38}$ Furthermore, the LDA-like treatment of local-field effects in our GWA model only approximates the screening across the highly inhomogeneous surface region. Our calculations refer to zero temperature and are performed with the theoretical lattice constant $(1.1 \%$ smaller than experiment), resulting in increased transition energies. Finally, the computations are performed for the ideal surface, neglecting defects and domain boundaries. Such imperfections are expected to induce distinct features in the optical anisotropy. ${ }^{13}$

\section{B. In-rich $\ln P(001)(2 \times 4)$}

Similarly to GaP, cation-rich $\operatorname{InP}(001)$ surfaces are characterized by $(2 \times 4)$ single-dimer reconstructions. ${ }^{33,39-41}$ Mixed-dimer $/ \delta$ structures (see Fig. 1) form for more/less Inrich surfaces. For anion-rich surfaces $\beta 2(2 \times 4)$ and $c(4$ $\times 4)$ reconstructions appear. ${ }^{33,42}$ The RAS spectra for the $(2 \times 4)$ reconstructed InP surfaces have recently been calculated ${ }^{16}$ and are in good qualitative agreement with the corresponding experimental results. ${ }^{42-44}$ This holds in particular for the stoichiometric trend, which was found to be very similar to the one obtained for GaP discussed above.

More recently, however, low-temperature RAS data for In-rich $\operatorname{InP}(001)(2 \times 4)$ surfaces, corresponding to the mixed-dimer structure, were measured ${ }^{45,46}$ (see Fig. 5). The features in the low-temperature spectrum are blueshifted with respect to the $300 \mathrm{~K}$ measurement, and sharpened. The negative anisotropy at low energies splits into peaks at 1.9, 2.1 and $2.6 \mathrm{eV}$ (denoted $S 1, S 2$, and $S 3$ ). The high resolution achieved experimentally renders the In-rich InP surface a particularly sensitive test case for our present study on the origin of RAS features. The fine structure measured at low temperatures was not resolved in our previous calculation. ${ }^{16}$ Furthermore, the energetic positions, in particular of the high-energy features, were not in accordance with experiment. We address these issues in the present work by using a much denser k-point mesh and including self-energy effects according to the GW approach discussed above.

The resulting spectra are shown in the upper part of Fig. 5. For low photon energies we obtain two pronounced nega-

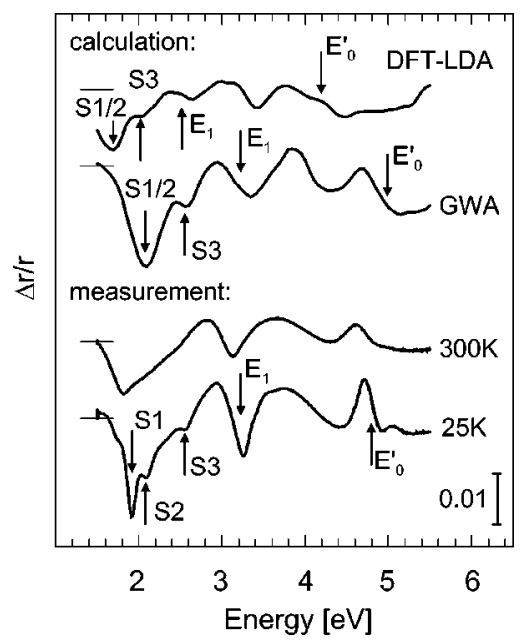

FIG. 5. RAS spectra $\left[\operatorname{Re}\left\{\left(r_{[110]}-r_{[110]}\right) /(r)\right\}\right]$ for the In-rich $\operatorname{InP}(001)(2$ $\times 4)$ surface. The calculated spectra were obtained within DFT-LDA and GWA. Measurements (Refs. 45 and 46) were performed at 300 and $25 \mathrm{~K}$. Bulk CP energies and surface-related features are indicated.

tive peaks (denoted $S 1 / 2$ and $S 3$ ). In the high-energy region features appear close to the $E_{1}$ and $E_{0}^{\prime}$ energies. They form a characteristic "three-buckle" shape. Separating spatially the contributions to the optical anisotropy from different slab regions, ${ }^{16,17}$ we find that $S 1 / 2$ and $S 3$ originate entirely from the uppermost four atomic layers. This is consistent with experiment, as the measured negative anisotropy for low energies is extremely stoichiometry dependent and therefore surface related. ${ }^{42-44}$ The features at the CP energies arise from transitions between bulk-like electronic states that are perturbed by the surface. In earlier calculations ${ }^{16,17}$ we have shown that these high-energy features are rather insensitive to the atomic surface structure. This agrees with experiment, ${ }^{42-44}$ where changes in the surface conditions had very little effect on the three-buckle shape.

The major experimental features are qualitatively reproduced in the DFT-LDA calculations, although at much lower photon energies. Inclusion of self-energy effects shifts the peak positions nonuniformly to higher energies, aligning them with experiment within a few tenths of an $\mathrm{eV}$. The shifts for the bulk related features are between 0.7 and 1.0 $\mathrm{eV}$, somewhat larger than the shifts of $0.4-0.5 \mathrm{eV}$ for the surface peaks. The changes of the line shape due to selfenergy effects distinctly improve the agreement with experiment.

In order to trace the origin of the surface-related anisotropies in detail, we extended earlier calculations of the $\operatorname{InP}(001)$ surface electronic structure ${ }^{47}$ to resonant surface states. We find four partially bound surface states, $V 1-V 4$, close to the VBM. $V 1$ is formed by the bonds between firstand second-layer cations, $V 2$ corresponds to the $\mathrm{P}$ dangling bond at the mixed dimer and $V 3$ arises from the second-layer In-In bonds at the anion site of the mixed dimer (cf. left panel of Fig. 6). V4 is complementary to $V 3$ and comparatively weakly localized at the second-layer cations at the cation site of the mixed dimer. The latter two states are degenerate with bulk bands for most parts of the SBZ (see also 




FIG. 6. (Left) Orbital character of surface states at $K$ of the mixed-dimer geometry of $\operatorname{InP}(001)(2 \times 4)$ (spacing $10^{-3} \mathrm{Bohr}^{-3}$ ). (Right) Calculated RAS due to transitions between these surface states.

Ref. 47). In addition, there are surface resonances. The strongly localized mixed-dimer bond $V 5$ forms a very flatband at about -0.8 to $-0.9 \mathrm{eV}$. The bonds between the uppermost $\mathrm{P}$ and the second-layer In atoms, $V 6$, disperse in energy between -1.3 and $-1.6 \mathrm{eV}$. The lowest unoccupied surface states, $C 1-C 3$, correspond to empty dangling bonds localized at the surface cations. $C 1$ is also localized at the mixed dimer (see also Fig. 5 in Ref. 16). Its energy agrees with the available inverse photoemission data. ${ }^{48}$ In order to gain an intuitive understanding of the optical anisotropy, we investigated the existence of surface state "signatures" by calculating the RAS due to transitions within pairs of the above identified surface states. We find that some of these transitions give rise to pronounced anisotropy features, the superposition of which accounts nearly for the low-energy part of the spectrum. Figure 6 shows the calculated contribu- tion to the RAS from transitions involving $V 1-V 5$ and $C 1-C 3$. This analysis provides a complete understanding of the surface features of the optical anisotropy: $S 1$ is caused by the bonds between first- and second-layer cations. $S 2$ arises from transitions mainly involving the second-layer In-In bonds. The symmetry break induced by the mixed dimer on the In-In bonds of the second atomic layer together with the dimer bond itself are responsible for $S 3$.

The calculated RAS in GWA reproduces the overall experimental data rather well. The distinct contributions from $V 1$ and $V 3$ at 2.0 and $2.2 \mathrm{eV}$ are not resolved however. Instead, we obtain one relatively broad minimum at $2.1 \mathrm{eV}$, due to the finite energy broadening necessary to account for the still limited number of wave vectors used to sample the SBZ. Apart from that, however, all experimentally found features can be identified and quantitatively described by slab calculations that take the electronic transitions within the uppermost atomic layers of the substrate into account.

\section{Hydrogenated Si surfaces}

As an example of step-induced optical anisotropies we study bilayer steps at the hydrogenated $\mathrm{Si}(111)$ surface. Since all dangling bonds are saturated, only intrinsic contributions to the RAS are expected. The two principal directions of miscut for the (111) surface are $\langle 11 \overline{2}\rangle$ and $\langle\overline{1} \overline{1} 2\rangle$. Assuming straight steps and barring any reconstruction, the first miscut (A in Fig. 7) gives step atoms with one dangling bond each, and the second with two dangling bonds each. In the latter case two different atomic structures are possible: a dihydride structure forms that either lies in a plane parallel (B1 in Fig. 7) or perpendicular to the step edge (B2 in Fig. 7). The optical response of these three configurations is investigated in the following. More precisely, adapting the accepted nomenclature for vicinal samples, the $\mathrm{Si}[3(111)$ $(11 \overline{2})]$ and $\mathrm{Si}[2(111)-(\overline{1} \overline{2} 2)]$ surfaces are studied, which consist of regularly spaced terraces that are three/two Si atoms wide along the $[11 \overline{2}] /[\overline{1} \overline{1} 2]$ direction, separated by single bilayer steps. In the following we refer to these structures as $\langle 11 \overline{2}\rangle$ and $\langle\overline{1} \overline{1} 2\rangle$ steps.

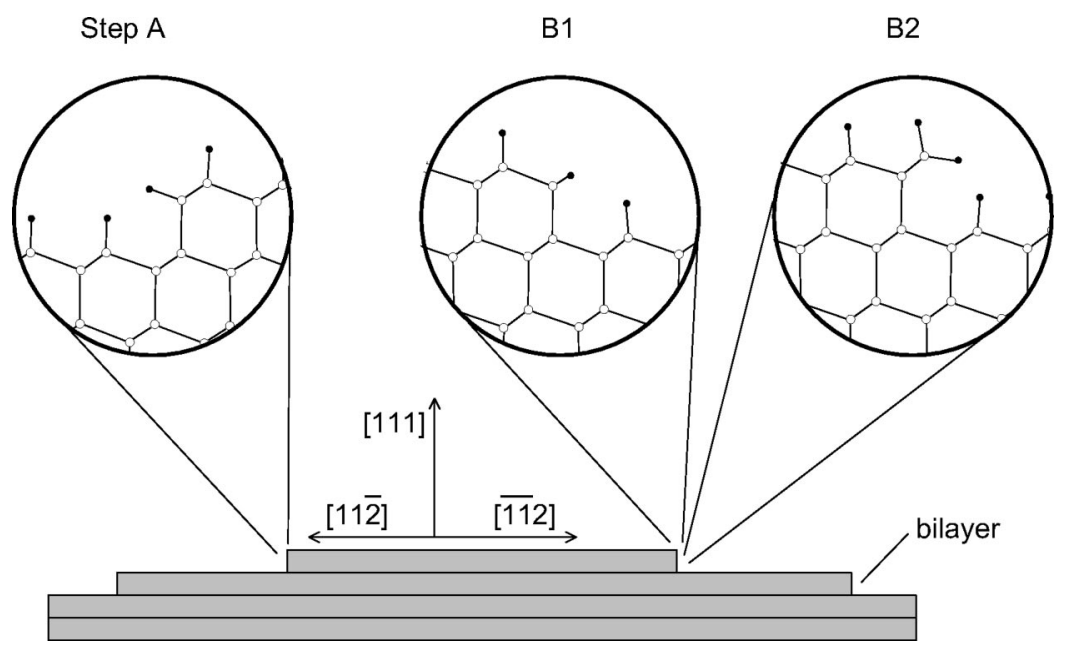

FIG. 7. Side view of the relaxed step configurations studied in this work. Empty (filled) circles represent $\mathrm{Si}$ (H) atoms. 




FIG. 8. Calculated RAS $\left[\operatorname{Re}\left\{\left(r_{\alpha}-r_{\beta}\right) /\langle r\rangle\right\}\right]$ for Si step geometries. The $\alpha$ and $\beta$ principal axes lie in the surface plane and are parallel and perpendicular to the respective step edge. The labels of the curves refer to the atomic structures of Fig. 7. Bulk CP energies are indicated.

The side views of the relaxed step geometries are shown in Fig. 7. All surface bonds are saturated by hydrogen. The $\mathrm{Si}$ atoms thus remain in bulk-like configurations and the relaxations are rather small and local. We find that the formation of a vertical dihydride structure in a plane perpendicular to the step edge (B2 in Fig. 7) is energetically preferred by $0.19 \mathrm{eV}$ per step atom compared to the $\mathrm{B} 1$ configuration. The formation of vertical rather than in-plane dihydride structures on $\langle\overline{1} \overline{1} 2\rangle$ steps is in agreement with infrared ${ }^{49-51}$ and Raman spectroscopy. ${ }^{52}$ The calculated RAS spectra for the three investigated step geometries are presented in Fig. 8. All steps give rise to strong anisotropy features close to the bulk $\mathrm{CP}$ energies. The main differences between the spectrum calculated for $\langle 11 \overline{2}\rangle$ and $\langle\overline{1} \overline{1} 2\rangle$ steps are a blueshift of about 0.1 $\mathrm{eV}$ and the occurrence of negative anisotropies at around 3 $\mathrm{eV}$ for the former and at around $4 \mathrm{eV}$ for the latter two spectra. Based on the striking similarity of the measured step-induced RAS to that of $\mathrm{Si}(110): \mathrm{H}$ it was suggested in Ref. 11 that the optical response of $\langle 11 \overline{2}\rangle$ steps arises from the (110)-like regions of the stepped surface. Figure 9 shows the spectrum for the $\mathrm{Si}(110): \mathrm{H}$ surface, calculated with an accuracy comparable to that obtained for the stepped surfaces. It is indeed rather similar to the RAS induced by $\langle 11 \overline{2}\rangle$ steps: maxima appear at the $\mathrm{CP}$ energies and there is an additional shallow minimum around $3 \mathrm{eV}$. The line shape, however, in particular around the $E_{1}$ energy, is different from that calculated for the step configuration and the relative ratio of the anisotropies at the $E_{1}$ and $E_{2} \mathrm{CP}$ energies is smaller. Furthermore, the overall similarity of the calculated RAS for all investigated step configurations suggests that one cannot explain the optical response of the $\langle 11 \overline{2}>$ stepped surface solely in terms of their (110)-like regions.

The overall similarity of all calculated step spectra indicates that the reflectance anisotropy originates from the per-

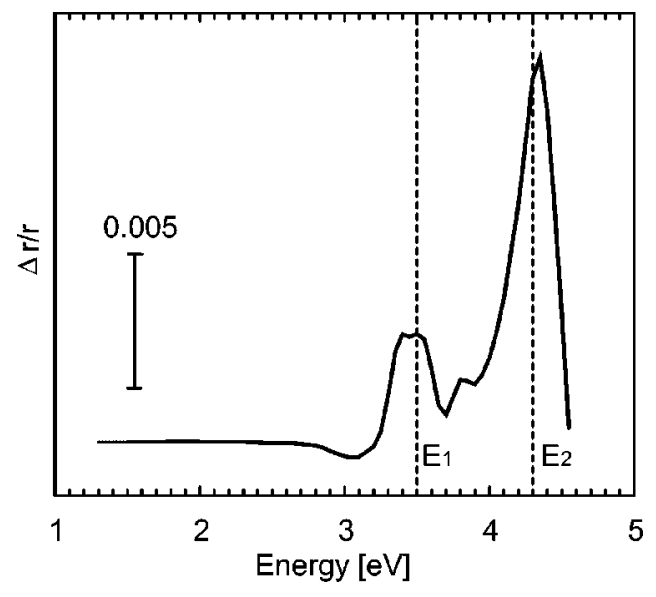

FIG. 9. Calculated RAS $\left[\operatorname{Re}\left\{\left(r_{[110]}-r_{[001]}\right) /<r>\right\}\right]$ for $\operatorname{Si}(110): \mathrm{H}$. Bulk CP energies are indicated.

turbation of bulk wave functions by the formation of surface steps, rather than from electronic transitions between steplocalized surface states. This picture is corroborated by our analysis separating spatially the contributions to the RAS (see Ref. 18): Electronic transitions within the uppermost 8 $\AA$ of the surface only modify the spectra, whose main features arise from transitions in deeper layers.

The calculated spectrum for $\mathrm{Si}(110): \mathrm{H}$ (Fig. 9) is interesting in its own right. It clearly resembles the imaginary part of the dielectric function of $\mathrm{Si}$. It is at variance with the tight-binding results of Ref. 53, but agrees rather well with experiment. ${ }^{11}$ In Ref. 53 it was concluded that the existence of $\mathrm{Si}$ and $\mathrm{H}$ vacancies at the $\mathrm{Si}(110): \mathrm{H}$ surface is a necessary condition for calculating line shapes in rough agreement with experimental data. Furthermore, it was stated that the surface local-field effect has to be included to obtain reasonable agreement with experiment. Our results, however, show that even an ideal $\mathrm{Si}(110): \mathrm{H}$ surface gives rise to a dielectric function-like RAS spectrum and do not indicate a particular importance of the surface local-field effect for that surface.

A direct comparison of the data calculated for stepped surfaces with experiment is not possible, as the existing measurements ${ }^{11}$ were performed on surfaces with considerably smaller step densities. Unfortunately, the lateral periodicities needed to accommodate wider terraces are still out of reach for $a b$ initio calculations of the optical response. In Ref. 11 it was noted, however, that the anisotropy signal for

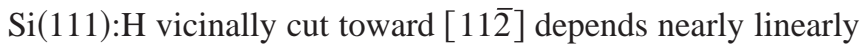
on the step density. A similar dependence was recently obtained for $\mathrm{Si}(001) .{ }^{13}$ Furthermore, our results, as well as earlier work, ${ }^{54}$ show that the atomic relaxations of the step edges are rather small and local. Therefore, it is expected that our calculations can be extrapolated to allow for a meaningful comparison with the measured data.

In Fig. 10 we show the experimental data ${ }^{11}$ for surfaces vicinally cut $\pm 5^{\circ}$. The upper curve in Fig. 10 shows the RAS of the surface cut toward $[11 \overline{2}]$. The main characteristics of the spectrum are the pronounced anisotropy maxima near the $E_{1}$ and $E_{2} \mathrm{CPs}$ of the bulk band structure. This is in 


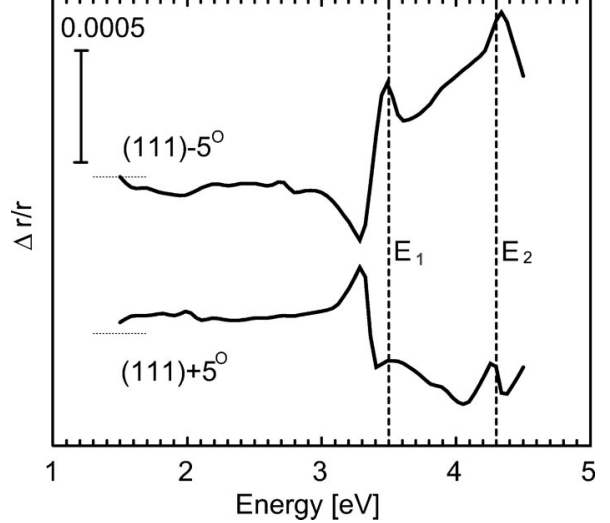

FIG. 10. Measured RAS (Ref. 11) for vicinally cut Si(111):H surfaces.

qualitative agreement with the corresponding calculated spectrum for $\langle 11 \overline{2}\rangle$ steps (A in Fig. 7). The measured anisotropies are about one order of magnitude smaller than the calculated values. This can be explained partially by the lower step density: a miscut angle of $\theta=5^{\circ}$ corresponds to an average terrace width $n$ of 11 rows $[\tan \theta=2 \sqrt{2} /(3 n$ $-1)$ ], as opposed to the width of three rows in our calculation. Temperature effects and sample imperfections neglected in our calculation also reduce the measured anisotropy signal. The calculated line shape, in particular around the $E_{1}$ energy, is only an approximate description of the measured signal. This is not surprising, however. The neglect of excitonic effects ${ }^{38}$ in the calculations leads to a bulk dielectric function where the $E_{1}$ peak is underestimated by about $50 \%$ and reduced to a weak shoulder of the $E_{2}$ peak. Since the step-induced reflectance anisotropy is to a large extent determined by transitions between bulk-like electronic states, the problematic description of the bulk dielectric function will cause differences in the line shape between the measured and calculated RAS spectra, in particular around the $E_{1}$ energy, even if no further approximations had been made in describing the surface. Additionally, the surface local-field effect, neglected in our study, may influence the line shape. ${ }^{53}$ If these limitations are borne in mind, however, the agreement between experiment and theory for the $\langle 11 \overline{2}\rangle$ stepinduced optical anisotropy is satisfactory.

This is seemingly not the case for $\langle\overline{1} \overline{1} 2\rangle$ steps. The lower curve in Fig. 10 shows data measured for the $\mathrm{Si}(111)$ :H surface miscut $5^{\circ}$ toward [ $\left.\overline{1} \overline{1} 2\right]$. Yasuda et al. ${ }^{11}$ assumed that the measured anisotropy is induced by bilayer $\langle\overline{1} \overline{1} 2\rangle$ steps. This interpretation does not agree with the calculations. Theory predicts positive anisotropy features at the $E_{1}$ and $E_{2}$ energies, while the measured anisotropies are negative. Even considering all the limitations discussed above, it is implausible that the calculations should yield a qualitatively wrong result. From Fig. 10 it seems as if the $(111)+5^{\circ}$ spectrum was an inverted and somewhat reduced version of the (111) $-5^{\circ}$ spectrum. This fact has already been noted in Ref. 11. By means of infrared ${ }^{49,55}$ and Raman spectroscopy, ${ }^{52}$ as well as scanning tunneling microscope (STM) measurements, ${ }^{56,57}$ it has also been demonstrated that the atomic structures of $\mathrm{Si}(111): \mathrm{H}$ surfaces miscut toward [1̄12] depend strongly on the preparation conditions. In particular, a transformation of straight dihydride-terminated $\langle\overline{1} \overline{1} 2\rangle$ steps into a staircase-like arrangement of monohydride terminated $\langle 11 \overline{2}\rangle$ steps was observed. These newly formed steps form angles of $60^{\circ} / 120^{\circ}$ with the original step edges. Assuming a complete transformation of straight $\langle\overline{1} \overline{1} 2\rangle$ steps into zig-zag chains of ideal $\langle 11 \overline{2}\rangle$ steps, the optical anisotropy of the surface should from pure geometrical considerations correspond to the anisotropy measured for straight $\langle 11 \overline{2}\rangle$ steps, but multiplied by $-\sqrt{3}$. In reality, the optical anisotropy of remaining, not-transformed parts of $\langle\overline{1} \overline{1} 2\rangle$ steps will partially cancel the signal from the zig-zag chains. A further disturbance of the "ideal" RAS spectrum can be expected from contributions due to the edges of the staircase structures. Thus, it seems likely that the optical spectrum of a $\mathrm{Si}(111)$ : $\mathrm{H}$ surface miscut toward [ $\overline{1} \overline{1} 2]$ is an inverted, reduced, and somewhat disturbed version of the spectrum measured for a surface miscut toward [112] . Obviously, the actual spectrum will depend on the preparation conditions. This hypothesis, if correct, gives a natural explanation of the experimental findings of Ref. 11, which are seemingly at odds with our calculations. However, further experiments are needed to verify the suggested interpretation.

\section{SUMMARY}

We have carried out extensive theoretical studies of the optical anisotropies of $(2 \times 4)$ reconstructed (001) surfaces of $\mathrm{GaP}$ and $\mathrm{InP}$, stepped $\mathrm{Si}(111): \mathrm{H}$, and hydrogenated $\mathrm{Si}(110)$ surfaces. The RAS spectra calculated for the compound semiconductor surface structures show characteristic "signatures" from surface states. In particular, transitions between cation-cation bonding states and empty cation dangling bonds give rise to strong negative anisotropies at low photon energies. In addition, we find features caused by surface-modified bulk wave functions. Surface-perturbed bulk wave functions are also mainly responsible for the pronounced anisotropy features close to the bulk CP energies caused by the formation of steps on $\mathrm{Si}(111)$ :H surfaces. Step orientation and geometry have little effect on the surface optical anisotropy. Inclusion of self-energy effects and a dense k-point sampling lead to a good agreement between slab calculations and experiments for the complete energy range of the spectrum. No long-range effects, such as photon-induced localization or quenching of bulk states, need to be invoked to explain the appearance of the surface optical anisotropy. The signal is mainly caused by optical transitions in the uppermost few atomic layers of the substrate.

\section{ACKNOWLEDGMENTS}

The authors thank David Aspnes, Norbert Esser, and Uwe Rossow for many stimulating and useful discussions. They 
also acknowledge grants of computer time from the John von Neumann-Institut Jülich, the Konrad-Zuse-Zentrum Berlin, and the DoD Challenge Program.

${ }^{1}$ Epioptics. Linear and Nonlinear Optical Spectroscopy of Surfaces and Interfaces, edited by J. F. McGilp, D. Weaire, and C. H. Patterson (Springer, Berlin, 1995).

${ }^{2}$ W. Richter, Philos. Trans. R. Soc. London, Ser. A 344, 453 (1993).

${ }^{3}$ D. E. Aspnes, Surf. Sci. 309, 1017 (1994).

${ }^{4}$ Z. Sobiesierski, D. I. Westwood, and C. C. Matthai, J. Phys.: Condens. Matter 10, 1 (1998).

${ }^{5}$ P. Chiaradia and R. Del Sole, Surf. Rev. Lett. 6, 517 (1999).

${ }^{6}$ F. Bechstedt, O. Pulci, and W. G. Schmidt, Phys. Status Solidi A 175, 5 (1999).

${ }^{7}$ L. Mantese, K. A. Bell, D. E. Aspnes, and U. Rossow, Phys. Lett. A 253, 93 (1999).

${ }^{8}$ K. Uwai and N. Kobayashi, Phys. Rev. Lett. 78, 959 (1997).

${ }^{9}$ V. L. Berkovits, P. Chiaradia, A. B. G. D. Paget, and C. Goletti, Surf. Sci. 441, 26 (1999).

${ }^{10}$ R. Del Sole and G. Onida, Phys. Rev. B 60, 5523 (1999).

${ }^{11}$ T. Yasuda, D. E. Aspnes, D. R. Lee, C. H. Bjorkman, and G. Lucovsky, J. Vac. Sci. Technol. A 12, 1152 (1994).

${ }^{12}$ L. Mantese, U. Rossow, and D. E. Aspnes, Appl. Surf. Sci. 107, 35 (1996).

${ }^{13}$ S. G. Jaloviar, J.-L. Lin, F. Liu, V. Zielasek, L. McCaughan, and M. G. Lagally, Phys. Rev. Lett. 82, 791 (1999).

${ }^{14}$ M. Fuchs and M. Scheffler, Comput. Phys. Commun. 119, 67 (1999).

${ }^{15}$ E. L. Briggs, D. J. Sullivan, and J. Bernholc, Phys. Rev. B 54, 14362 (1996).

${ }^{16}$ W. G. Schmidt, E. L. Briggs, J. Bernholc, and F. Bechstedt, Phys. Rev. B 59, 2234 (1999).

${ }^{17}$ A. M. Frisch, W. G. Schmidt, J. Bernholc, M. Pristovsek, N. Esser, and W. Richter, Phys. Rev. B 60, 2488 (1999).

${ }^{18}$ W. G. Schmidt and J. Bernholc, Phys. Rev. B 61, 7604 (2000).

${ }^{19}$ F. Aryasetiawan and O. Gunnarsson, Rep. Prog. Phys. 61, 237 (1998).

${ }^{20}$ Landolt-Börnstein, Numerical Data and Functional Relationships in Science and Technology (Springer, Berlin, 1982), Vol. III, p. 17a.

${ }^{21}$ J. E. Northrup, Phys. Rev. B 47, 10032 (1993).

${ }^{22}$ M. Rohlfing, P. Krüger, and J. Pollmann, Phys. Rev. B 52, 1905 (1995).

${ }^{23}$ R. Del Sole and R. Girlanda, Phys. Rev. B 48, 11789 (1993).

${ }^{24}$ L. Kipp, D. K. Biegelsen, J. E. Northrup, L.-E. Swartz, and R. D. Bringans, Phys. Rev. Lett. 76, 2810 (1996).

${ }^{25}$ M. Palummo, G. Onida, R. Del Sole, and B. S. Mendoza, Phys. Rev. B 60, 2522 (1999).

${ }^{26}$ O. Pulci, G. Onida, R. Del Sole, and L. Reining, Phys. Rev. Lett. 81, 5374 (1998)

${ }^{27}$ M. S. Hybertsen and S. G. Louie, Phys. Rev. B 37, 2733 (1988).

${ }^{28}$ F. Bechstedt, R. Del Sole, G. Cappellini, and L. Reining, Solid State Commun. 84, 765 (1992)
${ }^{29}$ G. Cappellini, R. Del Sole, L. Reining, and F. Bechstedt, Phys. Rev. B 47, 9892 (1993).

${ }^{30}$ R. Del Sole, Solid State Commun. 37, 537 (1981)

${ }^{31}$ F. Manghi, R. Del Sole, A. Selloni, and E. Molinari, Phys. Rev. B 41, 9935 (1990).

${ }^{32}$ S. Mirbt, N. Moll, K. Cho, and J. D. Joannopoulos, Phys. Rev. B 60, 13283 (1999).

${ }^{33}$ W. G. Schmidt, J. Bernholc, and F. Bechstedt, Appl. Surf. Sci. (accepted).

${ }^{34}$ P. A. Postigo, G. Armelles, T. Utzmeier, and F. Briones, Phys. Rev. B 57, 1359 (1998)

${ }^{35}$ M. Zorn, B. Junno, T. Trepk, S. Bose, L. Samuelson, J.-T. Zettler, and W. Richter, Phys. Rev. B 60, 11557 (1999).

${ }^{36}$ N. Sanada, S. Mochizuki, S. Ichikawa, N. Utsumi, M. Shimomura, G. Kaneda, A. Takeuchi, Y. Suzuki, Y. Fukuda, S. Tanaka, and M. Kamata, Surf. Sci. 419, 120 (1999).

${ }^{37}$ D. E. Aspnes and A. A. Studna, Phys. Rev. Lett. 54, 1956 (1985).

${ }^{38}$ S. Albrecht, L. Reining, R. Del Sole, and G. Onida, Phys. Rev. Lett. 80, 4510 (1998).

${ }^{39}$ C. D. MacPherson, R. A. Wolkow, C. E. J. Mitchell, and A. B. McLean, Phys. Rev. Lett. 77, 691 (1996).

${ }^{40}$ W. G. Schmidt, F. Bechstedt, N. Esser, M. Pristovsek, C. Schultz, and W. Richter, Phys. Rev. B 57, 14596 (1998).

${ }^{41}$ S. Mirbt, N. Moll, A. Kley, and J. D. Joannopoulos, Surf. Sci. 422, L177 (1999).

${ }^{42}$ K. B. Ozanyan, P. J. Parbrook, M. Hopkinson, C. R. Whitehouse, Z. Sobiesierski, and D. I. Westwood, J. Appl. Phys. 82, 474 (1997).

${ }^{43}$ D. Pahlke, J. Kinsky, C. Schultz, M. Pristovsek, M. Zorn, N. Esser, and W. Richter, Phys. Rev. B 56, R1661 (1997).

${ }^{44}$ M. Zorn et al. Appl. Phys. A: Mater. Sci. Process. 65, 333 (1997).

${ }^{45}$ T. Hannappel, S. Visbeck, M. Zorn, T. Zettler, and F. Willig, Appl. Phys. Lett. (submitted).

${ }^{46}$ W. G. Schmidt et al., Phys. Rev. B (in press).

${ }^{47}$ W. G. Schmidt and F. Bechstedt, Surf. Sci. 409, 474 (1998).

${ }^{48}$ S. Riese, E. Milas, and H. Merz, Surf. Sci. 270, 833 (1992).

${ }^{49}$ P. Jakob, Y. J. Chabal, K. Raghavachari, R. S. Becker, and A. J. Becker, Surf. Sci. 275, 407 (1992).

${ }^{50}$ S. Watanabe, Surf. Sci. 415, 385 (1998).

${ }^{51}$ S. Watanabe, Appl. Surf. Sci. 130-132, 231 (1998).

${ }^{52}$ M. A. Hines, Y. J. Chabal, T. D. Harris, and A. L. Harris, J. Chem. Phys. 101, 8055 (1994).

${ }^{53}$ B. S. Mendoza, R. Del Sole, and A. I. Shkrebtii, Phys. Rev. B 57, 12709 (1998).

${ }^{54}$ X.-P. Li, D. Vanderbilt, and R. D. King-Smith, Phys. Rev. B 50, 4637 (1994).

${ }^{55}$ P. Jakob and Y. J. Chabal, J. Chem. Phys. 95, 2897 (1991).

${ }^{56}$ G. J. Pietsch, U. Köhler, and M. Henzler, J. Appl. Phys. 73, 4797 (1993).

${ }^{57}$ J. Flidr, Y.-C. Huang, T. A. Newton, and M. A. Hines, Chem. Phys. Lett. 302, 85 (1999). 